\title{
Migrantes y medios en el ciberespacio: Construcción de identidad en los «no lugares»
}

\author{
Enrique Vaquerizo Domínguez
}

Recibido: 30.05.2019 - Aceptado: 25.06.2019

\section{Titre / Title / Titolo}

Les migrants et les médias dans le cyberespace: La construction

d'identité dans les «non-lieux»

Migrants and media in cyberspace: the construction

of identity in «non-places»

Migranti e media nel cyberspazio: la costruzione dell'identità nei «non luoghi»

\section{Resumen / Résumé / Abstract / Riassunto}

Este artículo estudia la relación entre los tres vértices del triángulo TIC, identidad y migraciones a través de un análisis lineal de los referentes de la temática. Un recorrido centrado en el escenario cada vez más móvil y dinámico en el que los tres actores interrelacionan, desde las rígidas fronteras geográficas tradicionales a la permeabilidad del transnacionalismo y el ciberespacio. La bibliografía consultada coincide en presentar a unas comunidades migrantes que experimentan en su cotidianeidad relaciones simultáneas y, en ocasiones, ambivalentes, con su territorio de origen y acogida, que diluyen construcciones identitarias asociadas a las fronteras de los Estados nación tradicionales dejando paso a nuevos significados y pertenencias dinámicos y cambiantes. El resultado señala la importancia de las Tecnologías de la información y Comunicación (TIC) como mediadoras, agrupando a migrantes geográficamente dispersas en la diáspora pero ahora intensamente conectadas, orbitando alrededor del territorio de origen. Un territorio recreado, reconstruido y en ocasiones idealizado de una manera simbólica.

Cet article étudie la relation entre les trois sommets du triangle TIC, identité et migrations à travers une analyse linéaire des référents du sujet. Un voyage centré sur le scénario de plus en plus mobile et dynamique dans lequel les trois acteurs sont en interaction, des frontières géographiques rigides traditionnelles à la perméabilité du transnationalisme et du cyberespace. La bibliographie consultée coïncide avec la présentation des communautés de migrants qui entretiennent des relations simultanées et parfois ambivalentes avec leur territoire d'origine et de réception, ces constructions identitaires diluées associées aux frontières des États-nations traditionnels, laissant la place à de nouvelles significations et appartenances dynamiques et changeantes. Le résultat souligne l'importance des technologies de l'information et de la communication (TIC) en tant que médiateurs, regroupant des migrants dispersés géographiquement dans la diaspora mais désormais étroitement connectés, en orbite autour du territoire d'origine. Un territoire recréé, reconstruit et parfois idéalisé de manière symbolique.
This article studies the relationship between the three vertices of the ICT triangle, identity and migrations through a linear analysis of the referents of the subject matter. A journey centered on the increasingly mobile and dynamic scenario in which the three actors interrelate, from the rigid traditional boundaries to the permeability of transnationalism and cyberspace. The bibliography consulted coincides with the presentation of migrant communities that experience simultaneous and, at times, ambivalent relationships with their territory of origin and reception, that dilute identity constructions associated with the borders of the traditional nation states, giving way to new dynamic and changing meanings and felings of belonging. The result points out the importance of Information and Communication Technologies (ICT) as mediators, grouping geographically dispersed migrants in the diaspora, who are now intensely connected, orbiting around the territory of origin. A recreated territory, reconstructed and sometimes idealized in a symbolic way.

Questo articolo studia la relazione tra i tre vertici del triangolo TIC, identità e migrazioni attraverso un'analisi lineare dei referenti del soggetto. Un viaggio incentrato sullo scenario sempre più mobile e dinamico in cui i tre attori si relazionano, dai rigidi confini geografici tradizionali alla permeabilità del transnazionalismo e del cyberspazio. La bibliografia consultata coincide nel presentare le comunità di migranti che vivono nella loro vita quotidiana relazioni simultanee, e talvolta ambivalenti, con il territorio di origine e di accoglienza, che diluiscono le costruzioni identitarie associate ai confini degli Stati nazionali tradizionali, lasciando il posto a nuovi significati e appartenenze dinamiche e mutevoli. Il risultato evidenzia l'importanza delle tecnologie dell'informazione e della comunicazione (TIC) come mediatori, che riuniscono migranti geograficamente dispersi nella diaspora ma ora intensamente collegati, e che ruotano intorno al territorio di origine. Un territorio ricreato, ricostruito e talvolta idealizzato in modo simbolico.

\section{Palabras clave / Mots-clé I Key words / Parole chiave}

Migración, TIC, diáspora virtual, transnacionalismo, ciberespacio. Migration, TIC, diaspora virtuelle, transnationalisme, cyberespace. Migration, ICT, virtual diaspora, transnationalism, cyberspace. Migrazione, TIC, diaspora virtuale, transnazionalismo, cyberspazio. 


\section{Introducción}

Las realidades de los colectivos inmigrantes en todo el planeta están hoy marcadas por los avances, en los medios de transporte y en las nuevas tecnologías de la comunicación. El aumento de las facilidades de desplazamiento y comunicación ha provocado que sean habituales los casos de comunidades procedentes de un mismo país o región que viven dispersas en diferentes lugares del globo pero manteniendo un contacto estrecho y una comunicación constantes. Este nuevo escenario, marcado por una tecnología que ha desdibujado las fronteras geográficas, económicas o políticas tradicionales, concede a los elementos culturales o identitarios un papel primordial a la hora de definir los sentimientos de pertenencia de los individuos.

La relación entre medios de comunicación y nuevas tecnologías de la información comenzó a ser estudiada principalmente por antropólogos y sociólogos desde la década de 1990, el enfoque tradicional se había centrado en la forma en que los colectivos migrantes utilizaban las tecnologías de la información, ya fuesen medios étnicos o generalistas para su asimilación o resistencia cultural desde el punto de vista de las sociedades receptoras y los efectos que este proceso provocaba en su grado de convivencia. El cambio de siglo y la generalización primero del uso de internet y la llegada más delante de las TIC situó el cambio de paradigma de los estudios migratorios, que comenzaron a prestar atención no sólo a la relación del sujeto migrante en la sociedad de acogida sino a los vínculos que conservaba con su territorio de origen.

Gracias, entre otros aspectos, al uso de las TIC, observamos fenómenos como el redescubrimiento, la asimilación y la reformulación de la cultura de origen por parte de migrantes de segundas o terceras generaciones que ni tan siquiera han conocido el país de procedencia de sus ancestros; o la creación de nuevas identidades híbridas entre individuos pertenecientes a dos países distintos, que usan los espacios virtuales como punto de encuentro.
Esta transformación ha cambiado la forma en que los migrantes se integran en sus países de destino, al mismo tiempo que modifican los lazos que mantienen con sus países de origen. Mientras que en el pasado, numerosos colectivos de migrantes se embarcaban en procesos acelerados de asimilación en sus nuevas sociedades de acogida, rompiendo en muchos casos los vínculos culturales originarios con el objeto de facilitar su integración, esos vínculos pueden perpetuarse hoy con mucha más facilidad.

\section{Enfoque teórico metodológico}

El marco teórico y metodológico para abordar dicho cambio de paradigma que utilizaremos en este artículo está ligado a un concepto: el de transnacionalismo. Tomaremos la noción de Vertovec (2009) para definir este proceso como «las actividades iniciadas y sostenidas por actores no institucionales, las cuales se organizan en grupos o redes de personas a través de las fronteras».

Bajo el enfoque transnacional de estudio de las migraciones, los migrantes desarrollan vidas móviles entre países de origen, destino y tránsito a través de un proceso migratorio, en ocasiones de ida y vuelta. Esta movilidad conectada les permitiría desarrollar simultáneamente actividades económicas, culturales, sociales y políticas, en sus sociedades de origen y de acogida, superando la rigidez de las fronteras nacionales y creando nuevos significados y pertenencias identitarias que tienen lugar más allá del espacio geográfico, a veces, como desarrollaré más adelante, en un territorio puramente virtual: el ciberespacio.

Desde la perspectiva del paradigma transnacional de estudio de las migraciones los territorios adquieren significado gracias a la acción de los sujetos. Son los propios migrantes quiénes diluyendo las fronteras han creado nuevos espacios en los que origen y destino se fusionan en un único territorio simbólico. Portes (2001) sostiene que el transnacionalismo engloba flujos humanos pero también del capital y la información. Del 
mismo modo este autor divide el transnacionalismo en dos variantes: una desde abajo impulsada por las motivaciones individuales de los individuos a la hora de migrar y otra desde arriba facilitada por las revoluciones experimentadas en los campos de la comunicación y el transporte. Sobre esta última incide la revolución de las TIC en las modificaciones de las relaciones y el espacio transnacional.

El concepto de transnacionalismo nace inicialmente para dar respuesta a las teorías migratorias que ponían el centro del debate en los aspectos económicos y en la asunción de que tras las etapas iniciales los colectivos migrantes se encaminaban inexorablemente hacia la asimilación dentro de la sociedad receptora.

Trabajos iniciales como los de Schiller (1992), al investigar a centroamericanos en Nueva York, evidenciaban, ya en las postrimerías del siglo XX, que los migrantes, lejos de asimilarse inevitablemente a la sociedad de acogida, continuaban en muchas ocasiones manteniendo relaciones económicas, políticas y sociales con sus lugares de origen. En el centro de esta visión de equilibrio entre dos mundos ya germinaba la idea de que, con el tiempo, los colectivos de migrantes interconectados llegarían a constituir comunidades transnacionales (Castro, 2005:1).

\section{Ciudadanos transnacionales: ruptura de la dicotomía entre asimilación y resistencia}

Profundizando en el caso de Estados Unidos, a partir de finales del siglo XX y principios del siglo XXI, los migrantes hispanos de Estados Unidos han experimentado un cambio en su actitud ante la integración en el país. Comienzan a mantener con más asiduidad su lengua materna y prácticas religiosas, conservan vínculos con sus sociedades de origen y se agrupan en comunidades activas culturalmente. En muchos casos, como señalan Arocena y Zina (2009:3), el objetivo ya ha dejado de ser americanizarse a toda costa aunque exista un sentimien- to de pertenencia estadounidense. Estos nuevos inmigrantes han desarrollado una doble identidad cultural o una identidad con un fuerte sentimiento de vinculación identitaria a los territorios de acogida y origen.

El aporte del transnacionalismo a las tesis multiculturalistas que propugnan la convivencia entre culturas separadas dentro de una misma sociedad, se basa en dos elementos fundamentales: la resignificación del territorio y el mantenimiento por parte de las culturas minoritarias representadas por comunidades migrantes de sus vínculos con las sociedades de origen. Un contacto que se conserva a través de las redes y conexiones entre territorios de origen y destino, creadas a partir de las migraciones internacionales. $Y$ unos vínculos que se materializan en base a diferentes dimensiones, que Arocena y Zina (2009:2) dividen en económica, política y cultural.

Si la primera de ellas se refiere a los fenómenos del envío de remesas y de las inversiones, la segunda a la participación electoral en la sociedad de origen o al ejercicio de la doble ciudadanía, la dimensión cultural se centraría en las asociaciones construidas por los migrantes para recrear y difundir sus expresiones culturales: música, religión, folclore, lengua, gastronomía o deportes.

A través de estas tres dimensiones, de la reformulación de su propia cultura en la sociedad de origen y del juego de espejos que el migrante percibe respecto a: 1) el autóctono y 2) a otros migrantes con los que comparte origen, es como el migrante transnacional construye su identidad (Moraes, 2008:9). Actualmente dicha identidad, se ve enriquecida y reformulada de nuevo al entrar en contacto a través de las redes digitales con nuevos migrantes con los que comparte origen que a su vez han migrado y residen en otros países.

Desde la perspectiva del transnacionalismo, asistimos por tanto a la resignificación del territorio como productor exclusivo de significados culturales, unos significados que son mutables en función del tiempo y de las circunstancias de las sucesivas generaciones migrantes que entran en escena. Como señala Castro (2005), las primeras generaciones de migrantes, mantendrían 
respecto a su sociedad de origen una interacción más intensa, en términos normativos y simbólicos, que los de segunda generación, para quiénes la relación con el territorio de origen cobraría una fuerza puramente simbólica y expresiva.

Sin embargo en el paradigma transnacional y desde el punto de vista de la construcción identitaria, el territorio no pierde completamente su importancia. De hecho, juega un papel insustituible, pero como referente no exclusivamente geográfico, sino histórico, social y simbólico. Desde la óptica de la experiencia migratoria, el lugar de destino se incorpora también con una importancia no desdeñable a esa construcción identitaria. Levitt y $\mathrm{Ny}$ berg-Sorensen (2004) sostienen que la migración transnacional «debe ser entendida como parte de dos o más mundos dinámicos interconectados» constituyendo un «proceso llevado a cabo por inmigrantes y sostenido por relaciones sociales multivinculadas que unen a sus sociedades de origen y destino». En cualquier caso, actualmente, al hablar de migración frente a los marcos multiculturales del siglo XX, hay que contar con un factor de aceleración en la evolución identitaria. Al intervenir en un mundo cada vez más interconectado, el migrante puede elegir entre diversas identidades «a la carta».

El cruce de fronteras y experiencias provoca esta resignificación del territorio y también una identidad socioterritorial cada vez más compleja y múltiple (Velasco, 1998:18). El espacio geográfico viaja con los migrantes y se manifiesta en objetos materiales, memorias y nostalgias compartidas. Ese espacio se hace presente a través de una dimensión puramente simbólica, con las mismas capacidades de producción cultural que la dimensión geográfica. Años después de estas primeras visiones transnacionales, las TIC han provocado que para muchos ciudadanos, y no solo migrantes, las fronteras sean un concepto obsoleto y cada vez más difuso.

La ciudadanía transnacional superaría el concepto tradicional de nación o comunidad originaria a la hora de gozar de plenos derechos políticos en la comunidad de origen y en la de acogida. En el sistema del Estado nación, los derechos ciudadanos siempre han ido aparejados a un concepto de nacionalidad, entendida como la pertenencia concreta a un estado. El auge de los movimientos migratorios internacionales y la generación de vínculos prácticos y simbólicos que superan ese marco clásico comienzan a cuestionar la identificación de los propios conceptos de nacionalidad y ciudadanía.

Como señala Ibáñez (2008:27) el crecimiento de la población inmigrante en todo el planeta contribuye a desarrollar una nueva dimensión de la identidad social y de los derechos ciudadanos en tanto que esta población introduce nuevos valores culturales, nuevas prácticas sociales, nuevas formas de lealtad política y de contradicción entre identidad y derechos y entre nacionalidad y ciudadanía. Este proceso no es exclusivo de los individuos. Cada vez existe un mayor número de entidades colectivas o grandes corporaciones que a pesar de haber dejado atrás sus países de origen, continúan ejerciendo sus derechos y manteniendo una influencia en la vida pública de estos de forma simultánea a la de acogida (Vilches, 2017:9). El espacio transnacional se desarrolla de esta forma como un fenómeno inherente al proceso de mundialización social, cultural y económico.

Junto al concepto de ciudadanía política transnacional, se puede hablar también de la ciudadanía social transnacional. Si la primera alude a las prácticas transnacionales que fortalecen los vínculos entre las personas y la comunidad en el espacio transnacional (entre las que se incluyen las prácticas de mantenimiento cultural e identitario), la segunda incluiría el derecho a ejercer influencia en los espacios de poder y participación cívica, como por ejemplo el derecho a voto en las elecciones, así como el desarrollo de otras prácticas políticas, como la participación en organizaciones de la sociedad civil y en organismos públicos.

\section{Las TIC y su influencia en la evolución del espacio transnacional}

Las Nuevas Tecnologías de la Comunicación y la Información, de ahora en adelante TIC, han supuesto un 
cambio importante acelerando y, en muchos casos, minimizando el alejamiento provocado por la distancia y el tiempo que los sujetos experimentan en todo proceso migratorio. Hoy estas herramientas permiten a las comunidades migrantes conservar con más eficacia aquellos usos sociales, símbolos y relaciones que las ayudan a configurar su identidad además de articular espacios de construcción política que pueden ser útiles para generar estrategias de denuncia y transformación social. De si estas comunidades utilizarán en el futuro esas herramientas solo para su propia reafirmación identitaria, o si lo harán también para incorporar usos e interacciones comunes e intercambiarlas con otras culturas diferentes, dependerá la convivencia en sociedades cada vez más diversas.

En este sentido, las TIC han posibilitado la evolución de un migrante desarraigado a un migrante más plenamente conectado. Como señala Melella (2016) su uso influye directamente en la construcción y expresión de las narrativas migratorias de los migrantes tanto en el plano individual como colectivo. Hoy los migrantes conservan vidas territorializadas además de mantener conexiones virtuales que les permiten construir su subjetividad y desarrollar su biografía

Desde este punto de vista las TIC juegan un papel fundamental dentro del proceso de transnacionalización del migrante y sus pertenencias identitarias, ya sean estas dobles o múltiples. De forma paralela al desarrollo exponencial de los movimientos migratorios experimentado durante las últimas décadas, se ha producido otro tipo de migración masiva que involucra también a miles de millones de personas. Hablamos de dos procesos paralelos: el aprendizaje y adopción e incorporación a la vida cotidiana del uso de las TIC y la ocupación del espacio digital por una parte de la humanidad a medida que se incrementaban los avances por el espacio físico.

La era de la información está marcada, como manifiesta Echeverría (1999), por la aparición de ese nuevo espacio social, que llamaríamos «el tercer entorno», caracterizado por herramientas digitales y electrónicas y que se uniría al primer entorno conformado por la naturaleza y el mundo rural y al segundo, representado por las ciudades. Este tercer entorno presenta una gran capacidad para fomentar las relaciones en red y a distancia entre las personas, independientemente de su presencia física. Se trata de una nueva migración que, en ocasiones, puede realizarse sin salir de casa, y que estaría determinada por un carácter más mental y emocional que físico.

De esta forma el desarrollo de TICs como las redes sociales ha desbordado los límites jurídicos, políticos y territoriales del Estado nación y relativizado la distinción entre lo próximo y lo lejano, ha reducido de forma significativa las distancias y desdibujado las fronteras de las culturas nacionales. Bermúdez y Martínez (2001) toman para explicar este proceso el concepto de «desanclaje» de Giddens (1990). A través de él escenifican como algunas culturas nacionales pierden en ese espacio virtual, el lazo orgánico que les ataba al territorio, e incluso a la lengua, alterando las formas tradicionales de entender las identidades nacionales.

\section{Nómadas digitales: Del transnacionalismo a la aparición de los no lugares}

De repente, y bajo estos parámetros, el espacio geográfico en el que se encuentren las personas ha dejado de ser determinante, solo se precisa una confluencia durante varias horas al día en un espacio electrónico junto a otros e-migrantes, como así los denomina (Echeverría, 1999). Años después de sus inicios la evolución de la red ha ido conformando conformando comunidades virtuales marcadas por un cierto grado de pertenencia basada en intereses comunes. Este «nomadismo digital» dentro del espacio electrónico, lejos de eliminar los movimientos migratorios sobre el espacio físico, se ha superpuesto sobre estos como un proceso paralelo y en ocasiones complementario. El ciberespacio provoca una representación cultural distinta del espacio territorial en el mundo real. Todo está dominado por la desterritorialización de los lugares, por la existencia de «nolugares» donde la tecnología suple a la presencia física. 
En la transición de un orden político internacional marcado por las fronteras nacionales a uno transnacional, tienen una responsabilidad fundamental la facilidad y frecuencia con la que los migrantes cruzan las fronteras y la deslocalización de los medios de comunicación tradicionales gracias a las nuevas tecnologías. Ambos factores están produciendo nuevas formas de imaginar la identidad y creando alternativas a aquellas marcadas tradicionalmente por el Estado nación.

Sobre ese papel de «comunidades imaginadas» en la relación con las estructuras de los Estados nación, Appadurai (1990) aporta el concepto de «esferas públicas diaspóricas», como elementos que acabarán por provocar la desaparición de los marcos anteriores. Según su análisis, estas «esferas diásporas públicas» se forjan en, y a través, de múltiples «paisajes» superpuestos: paisajes étnicos, paisajes financieros, paisajes tecnológicos o paisajes mediáticos e ideológicos. Estos paisajes están marcados por los flujos globales que, siguiendo su razonamiento, facilitan constructos identitarios e imaginarios transnacionales que hacen de la concepción del Estado tradicional un marco obsoleto.

En esas esferas se sitúan en muchas ocasiones los colectivos migrantes gracias a un contexto en el que la comunicación de masas se vuelve cada vez más determinada por herramientas electrónicas, nuevos canales que vinculan cada vez más a productores y audiencias a través de diferentes fronteras nacionales favorecidas por la migración. A medida que estas audiencias migrantes comienzan a interactuar entre los que se mudan y los que se quedan, se produce un número creciente de esferas públicas diaspóricas, vinculadas por una concepción de identidad cultural común.

\section{El Ciberespacio como punto de encuentro de la diáspora}

Las nuevas esferas públicas dejan, de esta forma, de ser patrimonio exclusivo de las naciones, para, a través de discursos mediados por los nuevos medios, ser detentados por colectivos sociales, religiosos o étnicos, orga- nismos profesionales o grupos de interés muy diversos entre sí. De esta forma el itinerario parece claro en la relación entre migrantes, territorio de acogida y origen: el espacio transnacional adquiere un carácter virtual y debilita las desconexiones físicas, es decir, se configuraría como territorio puente, punto intermedio y sin límites (Aliaga, 2016:7). El final de trayecto también: el «ciberespacio» como evolución del espacio transnacional y punto de encuentro del fenómeno diaspórico.

El primero en introducir el término de ciberespacio sería el escritor de Ciencia Ficción William Gibson en su novela Neuromante (1984), para designar el escenario recreado por las computadoras y sus interconexiones. A la definición conceptual de ese espacio puramente informático se fueron añadiendo los usuarios de la red y sus usos y formas alternativas de socialización para la apropiación social de las TIC. Existen más definiciones, relacionadas con la concepción del espacio virtual entre los usuarios de internet y otras redes telemáticas o de computadoras. Entre ellas, una que me parece especialmente sugerente: «El universo de redes digitales como un mundo de interacción y aventura, además de espacio de conflictos globales y una nueva frontera económica y cultural» (Levy, 2007: 46).

En general, existe bastante consenso para aceptar esa definición del ciberespacio como un mundo propio marcado por los contenidos culturales y la interacción humana mediado por artefactos de proceso informático y/o transmisión telemática de datos. Como manifiesta Soro (2006), el mayor ciberespacio del que disponemos sería el sistema abierto de internet. Alrededor de la red se articulan la mayor parte de interacciones humanas y la producción de flujos informativos y culturales en la actualidad.

La aparición de este escenario digital, motivado por el desarrollo de las TIC unido al crecimiento exponencial de los movimientos migratorios alrededor del mundo, ha favorecido la intercomunicación y el fortalecimiento de lo que denominaríamos el concepto de «diáspora virtual». Tötölyan (1991: 5) lo concreta como «una colectividad transnacional, separada y entrelazada a través de las fronteras de sus propios y otros esta- 
dos nación, manteniendo instituciones culturales y políticas». La diáspora engloba una red de comunidades de migrantes pertenecientes a distintos países y en cuya vinculación el factor cultural e identitario juega un papel de gran importancia. El desarrollo de los medios de comunicación ayuda a perpetuar los flujos migratorios entre determinadas zonas geográficas, a través de diferentes generaciones. Son esas redes asociativas que los migrantes establecen entre sus países de residencia y origen, las que, en muchos casos, mantienen funcionando el ciclo migratorio

Para Thieblemont-Dollet (2007), el término diáspora es más amplio y aludiría a las asociaciones de expatriados, refugiados, inmigrantes en el exilio o cualquier tipo de pertenencia étnica en general. Sobre todo resulta imposible entender las diásporas sin su carácter virtual, un carácter virtual que, como señala Tötölyan (1991), produce formas de identificación que escapan al contexto nacional y que surgen gracias a la mediación electrónica, los medios audiovisuales, la televisión por satélite y a internet. Todas estas mediaciones ofrecen distintas formas de vincular a los inmigrantes con sus países de origen. El ciberespacio constituye un espacio transnacional, en las que una serie de redes y herramientas se interconectan y permiten que a través de ellas fluyan ideas, prácticas y valores; aunque este flujo transnacional excede el movimiento físico e incluye, como hemos comentado anteriormente, a personas que no han participado en los propios procesos migratorios.

En el espacio digital y transnacional se construye una presencia conectada, en la que el migrante desarrolla una nueva forma de estar presente en su territorio de origen que sería más efectiva o emocional que puramente física. Las consecuencias del desarraigo, uno de los efectos consustanciales al proceso migratorio, quedan mitigadas al mantenerse un contacto permanente con la sociedad de origen, lo que provoca que el proceso migratorio resulte menos traumático una vez instalados los sujetos en el nuevo destino.

Por otra parte, el ciberespacio consigue vehicular gran parte de las interacciones de los colectivos migrantes de todo el mundo, produciendo un nuevo entorno que se alimenta de nuevas formas de comunicación. Los sujetos mantienen vivo el acervo cultural identitario de orígen, ya sea conservado íntegro o reformulado en entornos cada vez menos sólidos, donde la permanencia simbólica, sentimental y geográfica pueden coexistir cada vez más disociadas. Como hemos señalado al comienzo de este artículo el migrante puede hoy emprender la migración sin marcharse socialmente y construir su propia fórmula de vínculo cotidianos, culturales, y afectivos alternando entre el terreno físico y el virtual. El espacio virtual permite mantener elementos culturales a través de la interacción comunicativa con usuarios afines que le harán formar parte de dos entornos físicos a la vez.

Los sujetos integrados a través del entorno virtual experimentan interacciones comunicativas que les proveen de una nueva identidad espacial transnacional. Las culturas virtuales estarían compuestas por grupos de sujetos que se definirían por las formas, los usos y los contenidos de comunicación que establecen en el ciberespacio, al mismo tiempo estas prácticas modificarían también la propia identidad de esos sujetos. En este contexto, los modelos de integración y coexistencia cultural tradicionales como multiculturalismo, interculturalidad o transculturalidad comienzan a resultar un tanto rígidos para describir la realidad migrante. Al igual que las territoriales, las comunidades virtuales también crean sus propias reglas y códigos de interacción.

Estos códigos, como señalan Bermúdez y Martínez (2001), están basados en un predominio de lo visual, a través de los símbolos gráficos que les permiten transmitir emociones y que también constituyen elementos importantes en la configuración de la identidad de los habitantes de estos espacios desterritorializados. El desarrollo de estas comunidades, además, establece reglas de sanción y refuerzo que actúan como marco de integración de algunos miembros y de exclusión y diferenciación de los otros.

Nos encontramos ante una realidad paradójica, ya que si bien el aumento de los flujos migratorios, unido al avance en el terreno de las nuevas tecnologías de la información, está construyendo un mundo más peque- 
ño y móvil en el que las culturas e identidades colectivas tienden a des-territorializarse, las TIC ofrecen un nuevo espacio revirtiendo este proceso. Webs, blogs o redes sociales tienen la capacidad de ejercer un papel re-territorializador para esas identidades dispersas, reordenándolas a través de espacios virtuales comunes en los que millones de usuarios pueden encontrarse y definir quiénes son a través de un proceso de construcción simbólica.

\section{Recrear un país en el espacio digital}

El entorno digital permite a los colectivos migrantes pertenecientes a la diáspora recrear e inventar gracias a las comunidades virtuales «el espacio perdido de la nación». A pesar de no ser un tema aún demasiado explorado existen diversos estudios al respecto de la creación de identidad por parte de las diásporas virtuales. Moraes (2008), Komito (2011), Melella (2013) o Tsavkko García (2015).Todos coinciden en destacar la función del espacio virtual como catalizador y vehículo de expresiones de recreación de identidades en muchos casos mixtas y marcadas por un predominio de lo virtual.

Los migrantes tienen, en muchas ocasiones, en una página de Facebook, un blog o una web, el primer punto de contacto o información con el país al que emigrarán posteriormente y a la vez, el contacto más frecuente con sus países de origen una vez emprendido el proceso migratorio. Oiarzabal y Reips (2012) señalan que el sitio digital común creado por la interconexión de millones de ordenadores y personas ha pasado a ser un hogar virtual para muchas comunidades migrantes de todo el planeta.

Las herramientas y espacios pertenecientes a la Web 2.0 (redes sociales, foros, blogs, wikis) se convierten en el escenario donde recrean la cultura de sus países de origen, además de compartir sus propias experiencias comunes como emigrantes y las dificultades de adaptación en el país de acogida. Ese proceso se caracteriza por su dinamismo, ya que permiten una reinterpretación de la cultura de las sociedades receptoras a través de esas vivencias.
Si el correo electrónico en primera instancia, y herramientas como WhatsApp, Skype Telegram o Facebook posteriormente han reducido los costos de las comunicaciones de los migrantes, aumentando su frecuencia y enriqueciendo su contenido, estas herramientas digitales estarían cambiando además los procesos y las estructuras de la migración: principalmente debido al número de relaciones y contactos de migrantes que se encuentran en el extranjero, contactos que se producen entre ellos mismos y con sus sociedades de origen facilitando la aparición de nuevas identidades transnacionales (Komito, 2011:7). Es decir: individuos que se ven a sí mismos como miembros de un grupo nacional, cultural o étnico común a pesar del hecho de no vivir en las áreas geográficas asociadas a esas mismas naciones, culturas o etnias.

Ese papel como agentes en la conformación y reforzamiento de identidades entre las comunidades de migrantes hasta ahora había sido llevado a cabo en exclusiva por los medios de comunicación tradicionales, fundamentalmente a través de los llamados medios étnicos. Con la extensión del uso de las redes sociales y otras herramientas asociadas a la Web 2.0, la producción de la memoria de los países de origen se ha autonomizado con respecto a formas tradicionales e institucionalizadas mediáticamente.

En el conjunto de la población mundial se asiste a una eclosión de prácticas digitales, en las que la construcción de la memoria se pluraliza en cuanto a sus agentes y complejiza en cuanto a los lenguajes y modos de producción utilizados. Este tipo de prácticas resulta particularmente relevantes en el caso de las comunidades de migrantes que, de acuerdo con Ramírez Plascencia (2016), a partir de operaciones de selección y rescate de un pasado colectivo, activan referentes simbólicos, movilizan afectos y refuerzan sus vínculos identitarios con respecto a una patria común.

Parece que cada vez más las TIC son utilizadas de forma habitual por los migrantes para desarrollar, mantener y recrear redes personales, sociales, lingüísticas o culturales a nivel transnacional, de forma que para Oiarzabal (2012), el inmigrante, de ser un «inmigrante desarraigado», ha dado paso a conceptos como el «migrante online» 
(Nedelcu, 2009) o lo que Diminescu (2011) llama «inmigrante conectado»; un rol en el que el sujeto manifiesta sus conexiones con el país de origen a través de una presencia activa en canales de comunicación digitales.

Si las TIC han disminuido de forma importante el choque físico que se aprecia al cruzar una frontera. También han hecho lo propio con sus efectos culturales y emocionales, las consecuencias del desarraigo se diluyen al mantenerse la participación en la comunidad de origen, y al contacto con los sujetos que se han dejado atrás, así como con toda una serie de productos culturales: gastronómicos, musicales, audiovisuales o dinámicas y rituales de participación a los que los nuevos digitales proporcionan un acceso fácil y rápido:

Cada vez es más común que los migrantes mantengan relaciones remotas con características típicas de las relaciones de proximidad y para activarlas usan a diario el teléfono, internet o el correo electrónico. Esto hace que sea más fácil que antes quedarse cerca de la familia, saber lo que les está sucediendo, en casa o en otro lugar. La figura paradigmática del migrante desarraigado está cediendo paso a otra figura, una que todavía está no está definida, pero que corresponde a la de un migrante en movimiento que confía en tejer alianzas fuera de su propio grupo de pertenencia sin cortar sus vínculos con las redes de origen.

(Diminescu, 2008: 3)

$\mathrm{Al}$ proceso de apropiación de las nuevas tecnologías, por parte de las comunidades migrantes, ha contribuido durante los últimos años una disminución considerable de la brecha digital a medida que lo hacían el costo de acceso a internet y se multiplicaban los soportes tecnológicos: teléfonos móviles, tabletas, laptops, etcétera. No existe unanimidad sobre el grado de responsabilidad de la multiplicación de dispositivos en este proceso, ni en si la sociedad en red y las nuevas formas de organización transnacionales son una consecuencia determinada por los avances tecnológicos, o por el contrario, estos simplemente actúan como catalizadores de las nuevas necesidades y aspiraciones de las comunidades de migrantes en un mundo globalizado.

El desarrollo tecnológico es uno de los factores que han propiciado la sociedad en red, sin embargo el propio Castells (2001) señala que el contenido de un mensaje es indisociable de la forma en que este se transmite, lo que no significa necesariamente que el canal y sus implicaciones tecnológicas sean el único factor que lo determina. Internet ha cambiado la concepción espacio temporal y su simbología asociada, pero el agente de cambio no sería únicamente la tecnología, sino también la construcción de uso y sentido simbólico que se produce a través de ella y que en muchos casos existía de forma previa. En esta línea se manifiesta Melella (2016:16) citando a Tilly (2010) al señalar que las actuaciones que potencian las TIC pueden ser de carácter virtual o presencial. Las primeras tendrían la ventaja o la desventaja de no requerir un compromiso que incida fuera ciberespacio. Sin embargo, no hay que desconocer que los cambios en las acciones de grupos, movimientos sociales, colectivos migratorios, empresas, etcétera, no se deben tan sólo a la innovación tecnológica sino a la respuesta que esta proporciona a contextos y necesidades específicos

Siguiendo a Castells (2012: 87), «la tecnología no determinaría la organización social, sino que permitiría un tipo de organización que viene del deseo de cambio y que tendría más difícil llevarse a cabo sin internet».

En cualquier caso, parece evidente que el papel de las TIC para las comunidades migrantes ha excedido el de meras herramientas de comunicación para convertirse en mecanismos de producción simbólica y de afirmación cultural. Al extender la capacidad de participación, los nuevos canales digitales posibilitan como hemos señalado anteriormente, que la construcción de la memoria se pluralice. El agente de cambio no sería obligatoriamente el canal tecnológico en sí mismo, sino según Melella (2013), los usos e interacciones que este facilita. Por un lado, como espacio donde se desarrolla una cultura, pero además como artefacto cultural con propiedades contextualmente situadas.

Desde el punto de vista de las identidades construidas en el seno de las comunidades migrantes, ya sean diaspóricas, transnacionales, o posnacionales, éstas quedan definidas por su carácter híbrido y la superación de un marco que excede las fronteras del Estado nación. En cada escenario, esas identificaciones y diferenciaciones generan conflictos en los marcos de convivencia en 
que se desenvuelven y esa descripción de alteridad puede provocar según Melella (2013) una frontera simbólica y una reafirmación en sus «creencias singulares» como único camino de posicionamiento dentro de un contexto universal. Las identidades serían usadas por los grupos de migrantes para negociar con las alteridades con las que coexisten y reforzar sus vínculos como grupo.

Las culturas, no son elementos estáticos e inmutables; por el contrario, evolucionan constantemente y a distintos niveles, en muchas ocasiones siguiendo patrones lineales relacionados con la historia y la lengua. Internet cambia en gran medida estas reglas del juego al romper, de forma abrupta, los condicionantes espaciotemporales. A este proceso contribuye el hecho de que la pertenencia a una comunidad haya dejado de tener en muchos casos como referente principal el lugar de nacimiento, se puede ser reconocido como miembro de una comunidad aun sin haber nacido en ella o dejar de serlo pese a haber nacido en su interior.

En este aspecto, las nuevas tecnologías y canales de información además de ofrecer posibilidades de consumo mediático cada vez más amplias ayudan del mismo modo a configurar toda una arquitectura simbólica «a la carta», desligada del espacio de adscripción geográfica. Esa ruptura no tiene por qué producirse respecto a los rasgos culturales, y la identidad virtual se superpone en muchas ocasiones sobre la identidad previa, manteniendo y en algunos casos incluso potenciando, fortaleciendo y renovando sus elementos.

Si ejemplificamos el contexto de las redes sociales, observamos que éstas se configuran a través de usuarios que construyen perfiles públicos y se adscriben a comunidades junto a otros usuarios con los que comparten una determinada conexión. En esas comunidades se realiza una serie de interacciones, que se convierten en interpretaciones basadas como señala Turkle (1999) en el autorreconocimiento y la representación.

Esas representaciones se ejecutan en el entorno que se conoce popularmente como ciberespacio. Por ejemplo, si nos conectarnos a Facebook desde cualquier lugar del mundo, accedemos al espacio donde se encuentra nuestra comunidad y lo virtual cobra relevancia sobre lo geográfico. El ciberespacio, como señala Tsavko García (2015), se constituye como un lugar independiente y donde las relaciones sociales ocurren y se forman lazos sociales propios. Es la acción de los individuos en su proceso de selección e interacción en unos espacios virtuales determinados, la que reconfigura el ciberespacio y desarrolla una cibercultura específica.

Las TIC se constituyen como herramientas y escenarios para la construcción y la afirmación de identidades diaspóricas al extrapolar símbolos culturales e identitarios comunes a un nuevo plano. Páginas Web, blogs, wikis o redes sociales son, en su mayoría, y por su propia dinámica y funcionamiento, herramientas colectivas en las que el sentimiento de pertenencia y la actividad colectiva ocupa una gran importancia, y donde a través de las interacciones colaborativas que se producen en ellas se refuerza el proceso de construcción de identidades.

Entre el abanico de TIC que ofrece el panorama digital son especialmente las redes sociales las que, por sus grandes posibilidades comunicativas, permiten a los migrantes mantenerse en contacto con sus familiares y amigos, pero también sirven de escaparate de la expresión de lo inmaterial: sentimientos, ideas, y experiencias de los individuos. Podemos afirmar que las redes sociales y otros medios de comunicación digitales van configurando un espacio propio que supera los límites del territorio y que pasa a estar conformado por códigos culturales y simbólicos que construyen su identidad.

Tsavkko García (2015) afirma que estos medios se constituyen como un territorio situado entre lo local y lo global, donde los espacios offline y online convergen en un tercero en el que las relaciones directas y las realizadas a través de la red confluyen y se retroalimentan. La concepción geográfica y simbólica del territorio convive en muchas ocasiones en internet y a menudo espacio online contribuiría a ensanchar el geográfico expandiendo sus límites y configurando lo que Tsavkko García (2015) denomina como «el país digital». En su investigación remarca como los jóvenes vascos en la diáspora encuentran en Facebook un espacio que además de acercarlos al País Vasco, también los hacía sentir dentro de él a pesar de la lejanía. 
Como señalan Alonso y Oiarzabal (2010), las asociaciones de migrantes reinterpretan la cultura de las sociedades receptoras a través de sus propias vivencias, pero también recrean el espacio de su sociedad de origen, un espacio que al ser reinterpretado, de forma simbólica también sufriría cambios. Un proceso en el que ocupan un papel preponderante su pertenencia a la diáspora y los canales de comunicación utilizados para comunicarse con el resto de miembros que la componen.

Las herramientas comunicativas son aprovechadas principalmente por estos colectivos en una doble vertiente: por un lado agrupar a aquellos que comparten origen geográfico o cierta afinidad cultural o étnica en una sociedad de acogida y a la vez mantener los lazos con sus propias sociedades de origen. Redefinen conceptos clásicos como fronteras nacionales, espacio, identidad, tiempo y movilidad. Además esta creación de nuevos espacios también provoca a su vez la aparición de nuevos estilos de vida y formas de socialización en red lo que mejora la toma de decisiones de los migrantes generando nuevos hábitos transnacionales en el largo plazo (Nedelcu, 2012).

Pese a las posibilidades que parece abrir este horizonte el uso de las TIC por parte de los colectivos migrantes, aún ofrece panoramas disímiles en función de un amplio número de variables relacionadas con los capitales social, cultural y tecnológico que poseen los individuos. El capital económico, la escolaridad o el estatus legal aparecen algunos de los condicionantes más importantes para la utilización de las TIC. Del mismo modo en que es importante el uso que los migrantes hacen de estas herramientas, estos estarán condicionados por el peso del capital, conocimientos, limitaciones, habilidades y posibilidades de acceso con que se aproximan a ellas.

\section{A modo de conclusión}

Las TIC, han cambiado la forma en que los migrantes se integran en sus países de destino al mismo tiempo que el mantenimiento de los lazos que mantienen con sus países de origen. Vínculos que hoy pueden perpe- tuarse hoy con mucha más facilidad. El enfoque transnacional del estudio de las migraciones, establece que los migrantes desarrollan vidas móviles entre países de origen, destino superando la rigidez de las fronteras nacionales y flexibilizando los sentimientos de pertenencia identitaria

Desde la perspectiva del transnacionalismo, se pasa a una resignificación del territorio más simbólica que geográfica pero con una capacidad similar para crear productos culturales. Las TIC han acelerado la realidad transnacional, surgida a partir del incremento de los desplazamientos migrantes en el espacio físico, para llevar ese movimiento a un plano virtual. Los últimos años han visto como además del aumento de los flujos migratorios se producía otro tipo de migración masiva que involucra también a miles de millones de personas. A través del aprendizaje y adopción del uso de las TIC y la ocupación del espacio digital.

Este «nomadismo digital» dentro del espacio electrónico, lejos de eliminar los movimientos migratorios sobre el espacio físico, se ha superpuesto sobre estos como un proceso paralelo y en ocasiones complementario. El ciberespacio constituye un espacio transnacional y provoca una representación cultural distinta del espacio territorial en el mundo real. Todo está dominado por la desterritorialización de los lugares, por la existencia de «no-lugares» donde la tecnología suple a la presencia física.

En este contexto, para explicar las identidades de los individuos se han quedado obsoletos tanto el espacio del Estado nación como los modelos de integración y coexistencia cultural tradicionales como multiculturalismo, interculturalidad o transculturalidad. Al igual que las territoriales, las comunidades virtuales como espacios independientes también crean sus propias reglas y códigos de interacción específicos

De si las comunidades migrantes utilizarán en el futuro a las TIC solo para su propia reafirmación identitaria, o si lo harán también para incorporar usos e interacciones comunes a las sociedades que les acogen e intercambiarlas con otras culturas diferentes, dependerá la convivencia en sociedades cada vez más diversas. 


\section{Bibliografía}

AliagA, Felipe (2016), «Imaginarios en torno a la pertenencia nacional de los inmigrantes», Revista Latina de Sociología, 6, págs. 45-59. DOI: https://doi.org/10.17979/relaso.2016.6.1.1964.

Appadurai, Arjun (1990), Modernity at large: Cultural dimensions of globalization. Minneapolis: University of Minnesota Press. Retrieved from http://www.faculty.fairfield.edu/ dcrawford/appadurai.pdf.

Arocena, Felipe y Zina, Mariana (2011), «Migración, transnacionalismo y multiculturalismo. La vinculación de jóvenes uruguayos en Barcelona con su país de origen», Athenea Digital, 11(2), págs. 17-37. Recuperado de http:// cienciassociales.edu.uy/departamentodesociologia/arocena-felipe-zina-mariana-migracion-transnacionalismoy-multiculturalismo-la-vinculacion-de-los-emigradosuruguayos-en-barcelona-con-su-pais-de-origen.

Castro, Yerko (2005), «Teoría transnacional: revisitando la comunidad de los antropólogos», Política y cultura, 23, págs. 181-194. Recuperado de http://www.scielo. org. $\mathrm{mx} /$ scielo.php? script $=$ sci_arttext\&pid $=$ S018877422005000100011\&lng=es\&tlng=es.

Bermúdez, Emilia y Martínez, Gildardo (2001), «Los estudios culturales en la era del ciberespacio», Convergencia, Revista de Ciencias Sociales, 8(26), págs. 11-31. Recuperado de http://www.redalyc.org/pdf/105/10502601.pdf.

Castells, Manuel (2001), La era de la información. El poder de la identidad, vol. 2. Madrid: Alianza Editorial.

- (2012), Redes de indignación y esperanza: los movimientos sociales en la era de Internet, Madrid: Alianza Editorial.

Diminescu, Dana (2008), «The connected migrant: an epistemological manifesto», Social Science Information, 47, págs. 565-579. Recuperado de http://www.diaspora-stiintifica. ro/wp-content/uploads/2016/02/Dana-Diminescu_ Migrants-and-clandestinity.pdf.

ECHEVERríA, Javier (2010), Nomadismos contemporáneos: formas tecnoculturales de la globalización, Murcia: Universidad de Murcia, págs. 65-76. Recuperado de https://dialnet.unirioja.es/servlet/articulo?codigo $=3423487$.

Gibson, William (1984), Neuromante, Barcelona: Minotauro. GidDens, Anthony (1990), Consecuencias de la modernidad, Barcelona: Alianza Editorial.
IbÁÑEZ Angulo, Mónica (2008), De la identidad nacional a la ciudadania trasnacional: procesos migratorios y espacios trasnacionales. Procesos Migratorios y Espacios Transnacionales, Facultad de Humanidades y Educación Universidad de Burgos. Recuperado de http://docsgedime.files.wordpress. com/2008/02/monicaibanez.pdf.

Коміто, Lee (2011), «Social media and migration: virtual community 2.0. J. Am. Soc. Inf.» Sci. Technol, 62(6), págs. 1075-1086. Retrieved from https://onlinelibrary.wiley. com/DOI/pdf/10.1002/asi.21517.

LevitT, Peggy y Nyberg-Sorensen, Ninna (2004), «The Transnational Turn in Migration Studies», Global Migration Perspectives, 6, págs. 1-15. Recuperado de https://www. iom.int/jahia/webdav/site/myjahiasite/shared/shared/ mainsite/policy_and_research/gcim/gmp/gmp6.pdf.

LeVy, Pierre (2007), Cibercultura. La cultura de la sociedad digital, Barcelona: Antrophos. Recuperado de https://antroporecursos.files.wordpress.com/2009/03/levy-p-1997-cibercultura.pdf.

Melella, Cecilia (2013), Del inmigrante desarraigado al migrante conectado. La construcción de identidades de los colectivos andinos en la Argentina a través de la Web, VII Jornadas de Jóvenes Investigadores. Instituto de Investigaciones Gino Germani, Facultad de Ciencias Sociales, Universidad de Buenos Aires, Buenos Aires. Recuperado de https://www. aacademica.org/000-076/22.pdf.

- (2016), «El uso de las Tecnologías de la Información y Comunicación (TIC) por los migrantes sudamericanos en la Argentina y la Conformación de redes sociales», REMHU, Rev. Interdiscip. Mobil. Hum. 24 (46), págs. 77-90 Recuperado de http://www.scielo.br/scielo.php?pid=\$1980-8585 2016000100077\&script=sci_abstract\&tlng=es.

MoraEs, Natalia (2008), «La nación más allá del territorio nacional, Nacionalismo a distancia de migrantes uruguayos», Gazeta de Antropología, 24(1), págs. 2340-2792. Recuperado de https://dialnet.unirioja.es/servlet/articulo? codigo $=2581111$.

Nedelcu, Mihaela (2009), Le migrant online. Nouveaux modèles migratoires à l'ère du numérique. París: L'Harmattan.

Oiarzabal, Pedro J. (2012), «Diaspora Basques and Online Social Networks: An Analysis of Users of Basque Institutional Diaspora Groups on Facebook», Journal of Ethnic and Migration Studies, 38(9), págs. 1469-1485. DOI: 10.108 0/1369183X.2012.698216. 
Oiarzabal, Pedro J. y Reips, Ulf (2012), «Migration and Diaspora in the Age of Information and Communication Technologies», Journal of Ethnic and Migration Studies, 38, págs. 1333-1338. DOI: https:// DOI.org/10.1080/1369 183X.2012.698202.

Portes, Alejandro (2001), «Inmigración y metrópolis: Reflexiones acerca de la historia urbana», Migraciones Internacionales Año 1 (1), págs. 111-134. Recuperado de https:/ / www.redalyc.org/articulo.oa?id=15100106.

SCHILLER, Nina Glick (1992), Towards a Transnational Perspective in Migration: race class ethnicity and nationalism reconsidered, L. Bash \& C. Blanc-Staton (Eds.), Annals of the New York Academy of Sciences, vol. 645 Nueva York: New York Academy of Sciences, págs. 1-24.

Soro, Emilio (2006), Acción Comunicativa en el Ciberespacio: el análisis de las páginas web personales. Biblioteca online de Ciencias Da Comunicaçao. Covilha: Universidade de Beira Interior. Recuperado de http://www.bocc.ubi.pt/pag/ saez-soro-emilio-ciberespacio.pdf.

Thieblemont-Dollet, Sylvie (2007), Médias, migrations et cultures transnationales, Tristan Mattelart, dir., Médias, migrations et cultures transnationales, Bruxelles: De Boek, págs. 13-56. Recuperado de https://journals.openedition.org/questionsdecommunication/1918.
TÖlÖlYanK, Key (1991), «The Nation-State and its Others: In lieu of a preface», Diaspora. A Journal of Transnational Studies, 1, págs. 3-7. Recuperado de http://sites.middlebury.edu/nydiasporaworkshop/files/2011/04/D1-1-1991-TololyanKhachig-nationStateanditsOthersPreface.pdf.

Tsavkko García, Rafael (2015), «Transnacionalismo vasco e Internet: análisis del uso de blogs y Facebook en la Diáspora», Revista Cientifica Guillermo de Ockham, 13, págs. 59-67. Recuperado de http://revistas.usb.edu.co/index. php/GuillermoOckham/article/view/2065/1801.

VALdÉs, Juan Carlos y CABrera, Elizabeth (2013), Ciberespacio y cibersociedad, su relación con las formas alternativas de socialización para la apropiación social de las TICs, comunicación en II congreso sobre Tecnología, Educación y Sociedad, México. Recuperado de https://es.scribd.com/document/343828844/VALDES-Y-CABRERA-2013-Ciberespacio-y-Cibersociedad.

VelasCo, Laura (1998), «Identidad cultural y territorio: una reflexión en torno a las comunidades trasnacionales entre México y Estados Unidos», Región y sociedad, 11(15), págs. 105-129. DOI: https://doi.org/10.22198/rys.1998.15.a832.

Vertovec, Steven (2009), Transnationalism, London \& New York: Routledge. 


\section{IRENE VALLEJO \\ El infinito en un junco \\ La invención de los libros en el mundo antiguo}

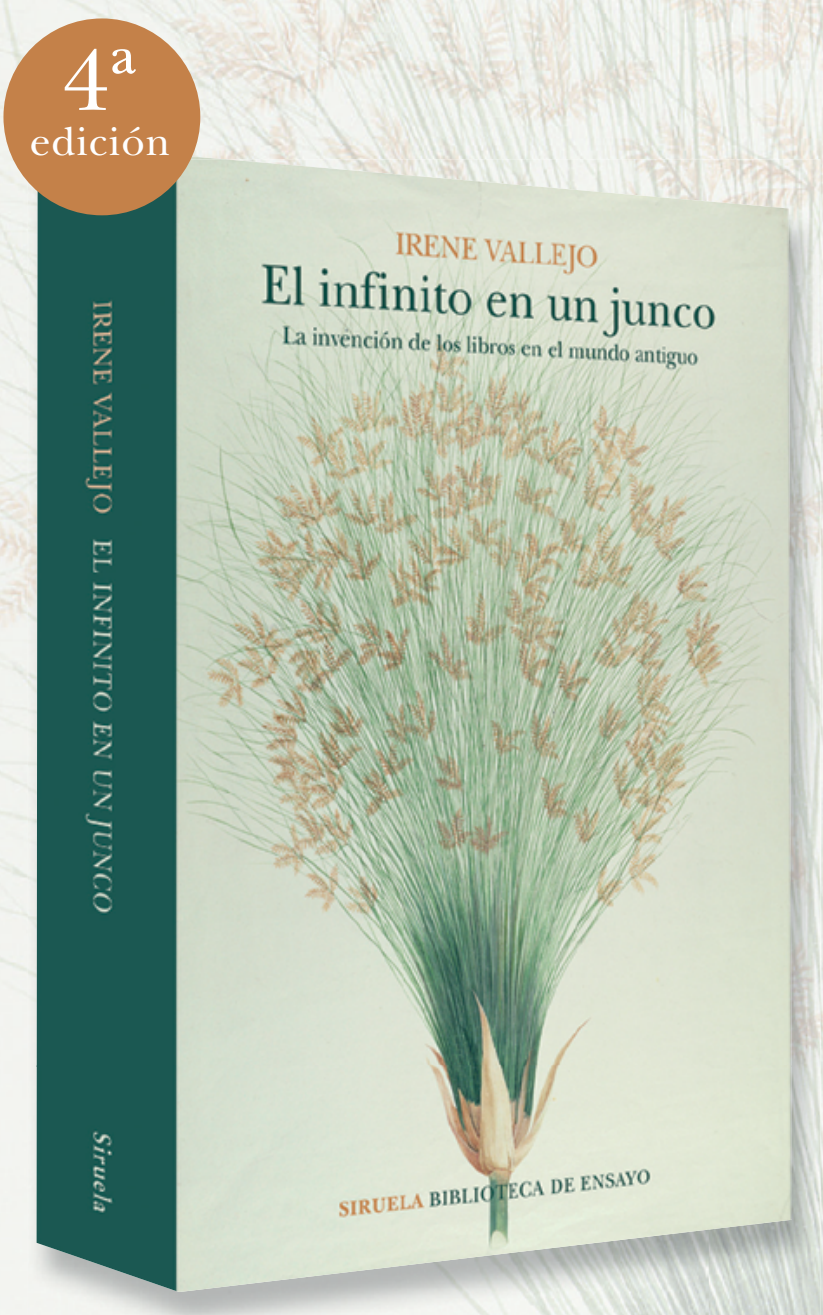

«Una admirable indagación sobre los orígenes del mayor instrumento de libertad que se ha dado el ser humano: el libro». Rafael Argullol

«Los libros de Irene Vallejo, claros e inteligentes, se leen muy bien e invitan a pensar. En la mejor línea humanista». Carlos García Gual

«Muy bien escrito, con páginas realmente admirables; el amor a los libros y a la lectura son la atmósfera en la que transcurren las páginas de esta obra maestra». MARIO VARgas Llosa

«Es un deleite leer la prosa de Irene Vallejo, creadora, brillante, plena de sensibilidad».

LUIS LANDERO 